\title{
Reducing the critical switching current in the free layer of magnetic random access memory
}

\author{
Jyh-Shinn Yang ${ }^{\mathrm{a}, *}$, Ching-Ray Chang ${ }^{\mathrm{b}}$ \\ a Institute of Optoelectronic Science, National Taiwan Ocean University, Keelung 202, Taiwan \\ ${ }^{\mathrm{b}}$ Department of Physics, National Taiwan University, Taipei 106, Taiwan
}

Available online 28 February 2006

\begin{abstract}
The switching behavior of synthetic antiferromagnetic (SyAF) bilayers in a toggle magnetic random access memory is investigated by micromagnetism. The strength of exchange coupling between the two sublayers significantly modifies the switching behavior of SyAF bilayers. The critical switching current decreases with decreasing the exchange coupling, and the low critical switching current can be achieved for SyAF bilayers solely based on the magnetostatic coupling. In addition, the optimization of the end shape in free layers and the ratio of width to height of conducting lines can further reduce the critical switching current.
\end{abstract}

(C) 2006 Elsevier B.V. All rights reserved.

PACS: 75.50.Mg; 75.40.Gb; 75.60.Jk; 85.75.Dd

Keywords: Micromagnetic simulation; Toggle-mode switching; Synthetic antiferromatic bilayer; MRAM

\section{Introduction}

Magnetic random access memory (MRAM) based on magnetic tunnel junctions is attractive because it has the combined attributes of non-volatility, high-speed operation, and infinite rewritability [1-3]. To realize a memory density compatible with the existing solid-state memory technology, the size of MRAM elements should be scaled down. As a result, the relatively high write-current threshold is required in the present MRAM design using the fieldinduced magnetic switching scheme. Hence, optimizing the write current of MRAM design is critically concerned. To this end, understanding the switching behavior of patterned memory elements is an inevitable task for developing a working MRAM. Recently, a synthetic antiferromatic (SyAF) bilayer was used as a free layer to suppress the bit-to-bit magnetostatic coupling and enhance the thermal stability $[4,5]$. To date, the works relevant to the switching properties of SyAF are done for the cases with uniform applied fields or the assumption of coherent

\footnotetext{
${ }^{*}$ Corresponding author. Tel.: + $886224622192 \times 6706$; fax: +886224634360

E-mail address: jyang@mail.ntou.edu.tw (J.-S. Yang).
}

magnetization rotation [6,7]. The present authors showed that to fully understand the writing process of memory elements, the effect of spatial non-uniformity of writing fields should be taken into account [9]. In this article, we shall study the switching behavior of SyAF bilayers of toggle MRAMs under the action of writing fields generated by the current-carrying conductor.

\section{Model}

We consider the memory element of a magnetic tunnel junction designed with an antiferromagnet, a pinned SyAF and a free SyAF. The free layer consists of two ferromagnetic layers, 1 and 2, that have the thickness $t_{1}$ $(=3.4$ or $3.2 \mathrm{~nm})$ and $t_{2}(=3 \mathrm{~nm})$, coupled through a nonmagnetic spacer with a thickness $=0.85 \mathrm{~nm}$ (Fig. 1a and b). Although the interlayer coupling depends on the details of the spacer layer, we here treat the AF exchange coupling strength as an adjustable parameter $J$ for simplicity. For the switching process, we only consider the SyAF free layer, whose shape is elliptical, oval or eye-shaped [8], used to study the effect of end shape. The magnetic material of the bilayer is a permalloy with representative material 


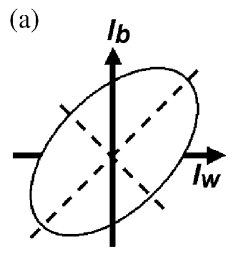

(b)

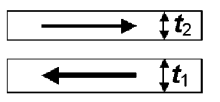

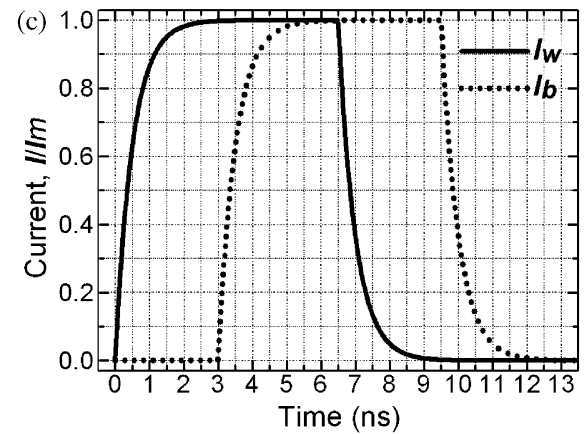

Time (ns)

Fig. 1. (a) Schematic of the Toggle-mode MRAM bitcell, (b) side view of SyAF free layers with the arrows indicating the initial orientation of magnetizations in sublayers, and (c) write-current pulse sequence in time used in the numerical simulation.

parameters as described in Ref. [9] except for damping constant $\alpha=0.05$. The length and width of the free layer are $420 \mathrm{~nm}$ and $315 \mathrm{~nm}$, respectively. Each sublayer is discretized into a two-dimensional array of $5 \mathrm{~nm} \times 5 \mathrm{~nm} \times t_{1,2}$ tetragonal meshes. The magnetization behavior of each mesh is numerically calculated by solving the Landau-Lifshitz equation [8]. The word and bit lines are oriented at $\pm 45^{\circ}$ with respect to the length direction of free layer, respectively, and lie beneath and above the free layer (Fig. 1a). A two-phase programming current pulse sequence, $I_{\mathrm{w}}$ and $I_{\mathrm{b}}$, is applied into the word and bit lines to switch the free layer according to the toggle-switching mode (Fig. 1c) [4]. To keep things simple, we assume that the conducting lines have the same dimensions with a rectangular cross-section, a width $w$ and a height $h$, and a separation of $30 \mathrm{~nm}$ from the free layer. The current pulses uniformly flowing through the bit and word lines are identical except a time delay of $3 \mathrm{~ns}$.

\section{Results and discussion}

As shown in Fig. 2, the critical switching current $\left(I_{\mathrm{sw}}\right)$ of the SyAF bilayer decreases with decreasing the exchange coupling strength between the two sublayers (Fig. 2). Also, a sharp end shape in combination with a small thickness difference of two sublayers can lead to a further reduction in the critical switching current. Note that the variation of critical switching current with the patterned shape is small in the absence of exchange coupling, in contrast to the results for a strong exchange coupling. Interestingly, the square of critical switching current as a function of exchange coupling can be well described simply by a quadratic polynominal. The origin of this behavior deserves a further investigation.

The interlayer AF coupling plays a vital role in the magnetic switching behavior of SyAF bilayers. For a strong exchange coupling, the magnetization directions of the two sublayers remain anti-parallel during the magnetic switching process (not shown here), resulting in a relatively high critical switching current. In contrast, for a bilayer with weak or null exchange coupling, the magnetization of

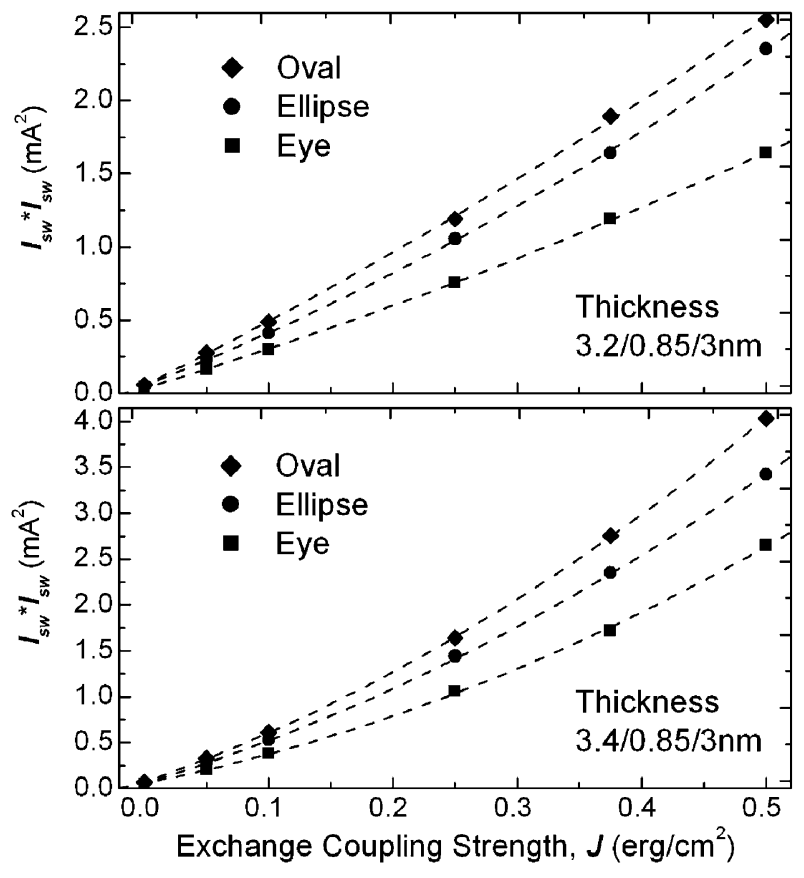

Fig. 2. Dependence of the critical switching current on the exchange coupling strength in toggle-mode MRAM. The dashed lines are the bestfitting curves by a quadratic polynominal. $w=150 \mathrm{~nm}$ and $h=350 \mathrm{~nm}$.

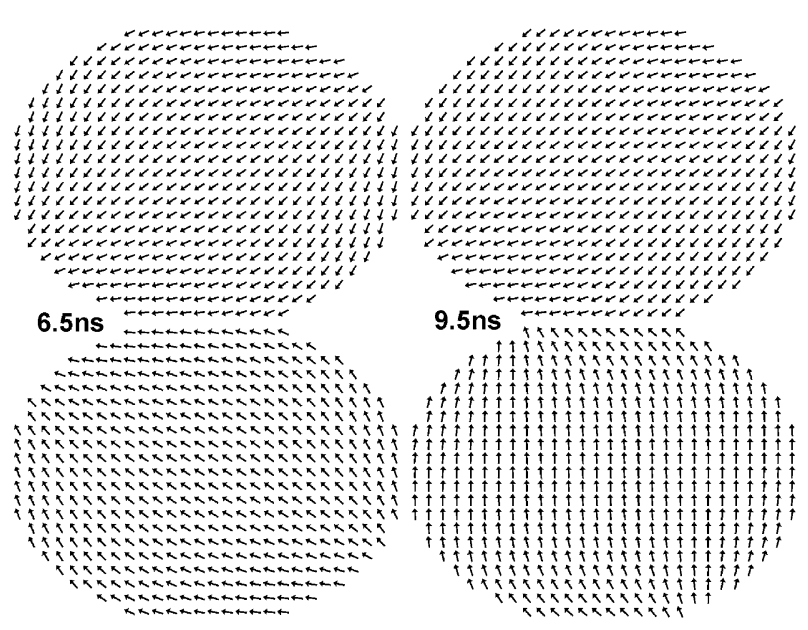

Fig. 3. The snapshot of transient states in the SyAF bilayer with an elliptical shape in the absence of exchange coupling during the magnetic switching process. The upper (lower) panels refer to the layers 2 (1) and the time of $6.5 \mathrm{~ns}(9.5 \mathrm{~ns})$ are the moments when the word (bit) currents are just shut off. $I_{\mathrm{sw}}=7.5 \mathrm{~mA}$ and $t_{1}=3.4 \mathrm{~nm}$. The values of $w$ and $h$ are the same as in Fig. 2.

one layer always switches earlier than the other owing to the thickness asymmetry of bilayers. The magnetostatic coupling from the switched magnetization will force the other one to rotate them toward the switched state with the aid of writing fields (see Fig. 3). Clearly, this kind of switching mode in SyAF bilayers can lower the switching current significantly, down to $6-8 \mathrm{~mA}$ for the present case.

Further optimizing the writing current can be achieved by adjusting the aspect ratio (AR) of width $w$ to height $h$ 


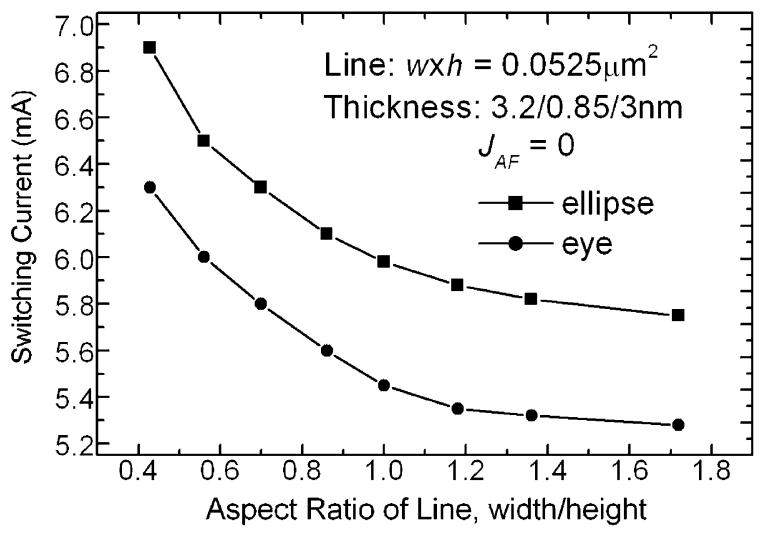

Fig. 4. Dependence of the critical switching current on the aspect ratio of conducting lines.

(AR) of conducting lines. Since increasing the AR of lines results in a decrease in the effective distance between the current source and the free layer, the writing field is enhanced; the critical switching current exhibits a monotonic decrease and gradually saturates as the $\mathrm{AR}$ of lines increases (Fig. 4).

In summary, we have utilized a micromagnetic approach to study the effect of exchange coupling, the patterned shape, the thickness asymmetry, and the AR of conducting lines on the critical switching current of SyAF bilayers. The present results demonstrate that a low critical switching current can be achieved in a toggle MRAM based on magnetostatically coupled bilayers by suitably selecting the geometric parameters.

\section{Acknowledgments}

This work was supported by the National Science Council of ROC under Grant NSC 93-2112-M019-006.

\section{References}

[1] J.M. Daughton, Thin Solid Film 216 (1992) 162.

[2] J.-G. Zhu, Y. Zheng, G.A. Prinz, J. Appl. Phys. 87 (2000) 6668.

[3] J. Åkerman, Science 308 (2005) 508.

[4] L. Savtchenko, A. A. Korki, B. N. Engel, N. D. Rizzo, J. A. Janesky, US Patent No. 6,545,906 B1, April 2003.

[5] V. Korenivski, D.C. Worledge, Appl. Phys. Lett. 84 (2004) 4559.

[6] J.-V. Kim, T. Devolder, C. Chappert, C. Maufront, R. Fournet, Appl. Phys. Lett. 85 (2004) 4094.

[7] D.C. Worledge, Appl. Phys. Lett. 84 (2004) 4559.

[8] X. Zhu, J.-G. Zhu, J. Appl. Phys. 93 (2003) 8376.

[9] J.-S. Yang, C.-R. Chang, W.C. Lin, D.D. Deng, IEEE Trans. Magn. $41(2005) 879$. 